\title{
Preliminary detection of the anti-tumour activity of indoline-2,3-dione derivative DH-12a targeting aminopeptidase $\mathbf{N}$
}

\author{
DE-XUAN CUI $^{1 *}$, XUE-JIAN WANG ${ }^{1,2^{*}}$, XIU-RONG ZHANG ${ }^{1}$, TING-KUN ZHAO ${ }^{1}$, XIU-XIANG GUO ${ }^{3}$, \\ SHUN-MEI LIU ${ }^{1}$, BING WANG ${ }^{2}$, WEN-QUAN MA ${ }^{2}$ and ZHI-QIN GAO ${ }^{1}$ \\ ${ }^{1}$ Pharmacy and Biological Science College, Weifang Medical University, Weifang, Shandong 261053; \\ ${ }^{2}$ Weifang High-tech Zone Biomedical Science and Technology Industrial Park, Weifang, Shandong 261205; \\ ${ }^{3}$ Sino-India Computer Software Institute, Weifang University of Science and Technology, \\ Shouguang, Shandong 262700, P.R. China
}

Received January 9, 2014; Accepted July 31, 2014

DOI: $10.3892 / \mathrm{mmr} .2014 .2552$

\begin{abstract}
Aminopeptidase N (APN) is important in tumour processes. The present study detected the anti-tumour activity of the novel APN inhibitor DH-12a, which is an indoline-2,3-dione derivative. In the present study, Bestatin, a clinical APN inhibitor was used as a positive control. The expression of APN in the ES-2 and $3 \mathrm{AO}$ cell lines were assessed using flow cytometry and the drug inhibition constants of DH-12a $\left(\mathrm{K}_{\mathrm{i}}=13.15 \mu \mathrm{M}\right)$ and Bestatin $\left(\mathrm{K}_{\mathrm{i}}=16.57 \mu \mathrm{M}\right)$ were assessed using a double reciprocal method of competitive inhibition. The in vitro effects of $\mathrm{DH}-12 \mathrm{a}$ on cell proliferation were assessed using a 3-(4,5-dimethyl-thiazol-2-yl)-2,5-diphenyl tetrazolium bromide assay on human cell lines of ES-2 $\left(\mathrm{IC}_{50}=43.8 \mu \mathrm{M}\right)$, A549 (inhibition rate $=41.5 \%$ at $160 \mu \mathrm{M} \mathrm{DH}-12 \mathrm{a}$ ), HL60 (inhibition rate $=47.83 \%$ at $160 \mu \mathrm{M} \mathrm{DH}-12 \mathrm{a})$ and $3 \mathrm{AO}\left(\mathrm{IC}_{50}=70.2 \mu \mathrm{M}\right)$. The inhibition rates were consistently higher than those of Bestatin. The effects of DH-12a on cell migration (inhibition rates in ES-2 cells and 3AO cells were 56.4 and $76.5 \%$, respectively at $15 \mu \mathrm{M}$ ) and invasion (inhibition rates in ES-2 cells and $3 \mathrm{AO}$ cells were 75.6 and $66.5 \%$, respectively at $15 \mu \mathrm{M}$ ) were assessed using transwell plates. The in vivo effects of $\mathrm{DH}-12 \mathrm{a}$ on tumour proliferation and lung tumour metastasis were determined using an H22 xenograft mice model, where DH-12a was administered in combination with genotoxic 5-fluorouracil. The anti-tumour activities of $\mathrm{DH}-12 \mathrm{a}$ in vivo were also greater than
\end{abstract}

Correspondence to: Mrs. Zhi-Qin Gao or Mr. Xue-Jian Wang, Pharmacy and Biological Science College, Weifang Medical University, Weifang, Shandong 261053, P.R. China

E-mail: zhiqingao2013@163.com

E-mail:wxj2901@126.com

*Contributed equally

Key words: aminopeptidase $\mathrm{N}$, indoline-2,3-dione derivative, migration, invasion those of Bestatin. In conclusion, the in vitro effects of DH-12a on tumour proliferation, migration and invasion were consistent with the in vivo effects. In addition, $\mathrm{DH}-12 \mathrm{a}$ exhibited greater anti-tumour properties compared with Bestatin.

\section{Introduction}

Aminopeptidase N (APN; EC 3.4.11.2; CD13), also termed CD13, is a type 2 transmembrane zinc-dependent metallopeptidase of the superfamily of gluzincins $(1,2)$. APN is expressed in numerous cells and tissues and is highly expressed in certain types of tumour, where it functions in tumour progression (3). APN expression may be involved in the invasion and metastasis of cancer (4-7). Therefore, APN has been used as a new prognostic marker of cancer.

Bestatin is a dipeptide with a low molecular mass that functions as an APN inhibitor. It is also the only inhibitor of APN that has been used clinically as an adjuvant drug in the treatment of cancer, including leukaemia and ovarian carcinoma $(8,9)$. However, the inhibition of APN by Bestatin is non-specific and exhibits lower efficacy and significant toxicity in the bone marrow and the gastric and intestinal mucosa $(10,11)$. Therefore, it is necessary to identify new APN inhibitors with a low toxicity and high specificity.

DH-12a, a compound synthesised by Jin et al (12), is more efficient at inhibiting the activity of APN than Bestatin. The structure of these two compounds is shown in Fig. 1. In the present study, the possible functions of DH-12a in tumour progression were examined. As inhibition of APN/CD13 has been observed to suppress the progressive potential of ovarian carcinoma (OVCA) cells (13), the OVCA cell lines ES-2 and $3 \mathrm{AO}$ were predominantly selected for in vitro investigation. Initially, APN in the ES-2 and 3AO cell lines were quantified. Using Bestatin as a positive control drug, the ability of DH-12a to inhibit the activity of APN and the inhibition constant of DH-12a to APN were determined. Subsequently, with Bestatin treatment as a positive control, the effects of DH-12a on cell proliferation were examined in the ES-2, A549, HL60 and 3AO cell lines using an MTT assay. The effects on cell invasion 
and cell migration were assessed in the ES-2 and $3 \mathrm{AO}$ cell lines using a Transwell plate. The anti-tumour activity of DH-12a was also determined in vivo, where DH-12a or Bestatin was combined with the genotoxic chemotherapeutic 5-fluorouracil (5-Fu).

\section{Materials and methods}

Cells, reagents and animals. The human ovarian clear cell adenocarcinoma cell line ES-2, human alveolar epithelial cell line A549, human promyelocytic leukemia cell line HL-60 and human ovarian carcinoma cell line $3 \mathrm{AO}$ were obtained from the Cell Bank of Shanghai (Shanghai, China) and maintained in RPMI-1640 (Thermo Fisher Scientific, Inc., Beijing, China) supplemented with $10 \%$ newborn calf serum (NCS; Zhejiang Tianhang Biotechnology Co., Ltd., Zhejiang, China). The cells were incubated at $37^{\circ} \mathrm{C}$ in a humidified atmosphere containing $5 \% \mathrm{CO}_{2}$. The APN/CD13 mouse-anti-human antibody (CD13mAb clone WM15) was purchased from BD Pharmingen (San Diego, CA, USA. Four to five-week-old female Kunming mice were purchased from the Experimental Animal Center of Weifang Medical University (Weifang, China). The procedures were performed in accordance with the Guidelines of the Animal Care and Use Committee at Weifang Medical University.

Reagents. Trypsin, 3-(4,5-dimethyl-thiazol-2-yl)-2,5-diphenyl tetrazolium bromide (MTT), dimethyl sulfoxide (DMSO), 5-Fu and 1-leucine-p-nitroanilide (cat. no. L9125) were purchased from Sigma-Aldrich (St. Louis, MO, USA). Basement membrane matrix (Matrigel, cat. no. 356234) was purchased from BD Biosciences (Franklin Lakes, NJ, USA), and DH-12a, an indoline-2,3-dione derivative, was provided by the Department of Medicinal Chemistry, School of Pharmaceutical Sciences, Shandong University (Jinan, China).

Flow cytometric analysis. Fluorescence-activated cell sorting (FACS) was performed to quantify the levels of APN expression on the surface of the ES-2 and 3AO cells. This was determined using methods described previously (3). Cells were incubated with a APN/CD13 mouse-anti-human monoclonal antibody (BD Pharmingen) for $30 \mathrm{~min}$ at $4^{\circ} \mathrm{C}$ in the dark. At the same time, the blank control group was administered with the same treatment with the exception of the monoclonal antibody. Subsequently, the cells were washed twice with phosphate-buffered saline (PBS) and then resuspended in PBS and analysed using a FACScan flow cytometer (FACSCalibur; Becton-Dickinson, San Jose, CA, USA) using Cell Quest software (BD FACSDiva 6.0; Becton-Dickinson). The levels of APN/CD13 were estimated as mean fluorescence intensity following subtracting those of the blank control group cells (14).

Enzyme activity assay. The activity of APN/CD13 on the surface of the ES-2 cells was determined using the methods described previously (12). A cell suspension was prepared and then washed with PBS. Cells $\left(5 \times 10^{5}\right)$ were then suspended in PBS in each well of a 96-well plate containing various concentrations of Bestatin or DH-12a, with a final volume of $200 \mu \mathrm{l}$. The working concentrations of the two drugs were set as 2.5, 10, 40, 160 and $640 \mu \mathrm{M}$. Following incubation with $1.6 \mathrm{mM}$ l-leucine-p-nitroanilide for $30 \mathrm{~min}$ at $37^{\circ} \mathrm{C}$, the enzyme activity was estimated by measuring

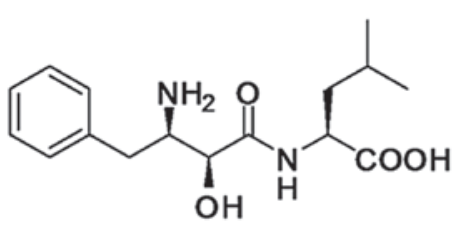

Bestatin

MW: 308.17

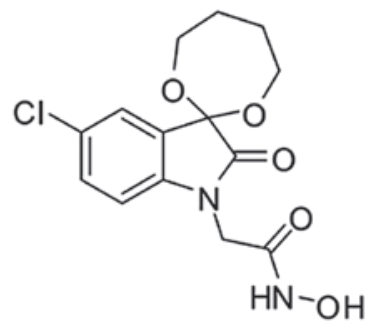

DH-12a MW: 326.73
Figure 1. Chemical structure of aminopeptidase $\mathrm{N}$ inhibitors.

the absorbance at $405 \mathrm{~nm}$ using a microplate reader (Model 680; Bio-Rad, Hercules, CA, USA). The APN activity inhibition rates of the compounds were calculated using the following formula: (ODcontrol - ODtested) / ODcontrol x 100\%, where OD was the mean value of three replicate wells.

Assessment of the inhibition constant $\left(K_{i}\right)$. Determination of $\mathrm{K}_{\mathrm{i}}$ was constructed using the kinetics of aminopeptidase inhibition, as described previously (3). ES-2 cells ( $\left.1 \times 10^{5}\right)$ intensely expressed APN/CD13 and different concentrations of inhibitors $(1,2,3,4$ and $5 \mu \mathrm{g} / \mathrm{ml})$ were incubated with two concentrations of substrate $(3.2 \mu \mathrm{M}$ and $6.4 \mu \mathrm{M})$ at $37^{\circ} \mathrm{C}$, respectively. The reaction velocities were measured by monitoring the increasing absorbance at $405 \mathrm{~nm}$ every $1 \mathrm{~min}$ for $15 \mathrm{~min}$. $\mathrm{K}_{\mathrm{i}}$ was calculated using the following equation:

$$
\frac{1}{v}=\frac{K_{m}}{V_{\max [S]}} \times \frac{[I]}{K_{i}}+\frac{K_{m}}{V_{\max [S]}}+\frac{1}{V_{\max }}
$$

where $[I]=$ concentration of APN inhibitor. Under two concentration ranges of $[S],[I]=-\mathrm{K}_{\mathrm{i}}$ at the point of intersection of $1 / \mathrm{v}$, vs. $[I]$.

Cell proliferation assay. The cell proliferation assay was performed using methods described previously (15). Cells (2,000 each) of the HL60, A549, ES-2 and 3AO cell lines were seeded into a 96-well plate and allowed to grow for $20 \mathrm{~h}$. The cells were then treated with various concentrations of Bestatin $(20,40,80,160,320$ and $640 \mu \mathrm{M})$ or DH-12a $(5,10,20,40$, 80 and $160 \mu \mathrm{M})$ for a further $48 \mathrm{~h}$ at a final volume of $200 \mu \mathrm{l}$. An MTT assay was performed by adding $20 \mu \mathrm{l}$ MTT $(5 \mathrm{mg} /$ $\mathrm{ml}$ ) to each well followed by incubation for an additional $4 \mathrm{~h}$. Subsequently, the culture was removed and $200 \mu \mathrm{l}$ DMSO was added to dissolve the formazan crystals. The light absorbance of the solution at $490 \mathrm{~nm}\left(\mathrm{OD}_{490}\right)$ was measured using an enzyme-linked immunosorbent assay reader (Model 680; Bio-Rad) and $\mathrm{OD}_{630}$ was measured as a reference. The inhibitory rates of the APN inhibitors were calculated using the following formula: (ODcontrol - ODtested) / ODcontrol x 100\%, where OD represents the mean value of three replicate wells. The $\mathrm{IC}_{50}$ value was determined using Origin 7.5 software (OriginLab Corporation, Northampton, MA, USA).

In vitro migration assay. The inhibitory effect of DH-12a and Bestatin on the cell migration of the ES-2 or $3 \mathrm{AO}$ cell lines was evaluated in 24 -well transwell plates $(8 \mu \mathrm{m}$ pore 
size; Corning Costar, Corning, NY, USA). Based on preliminary experiments, the cells were incubated in 6-well plates. Subsequently, $1 \times 10^{5} \mathrm{ES}-2$ or $3 \mathrm{AO}$ cells, which had been incubated with $15 \mu \mathrm{M}$ DH-12a or $320 \mu \mathrm{M}$ Bestatin for $48 \mathrm{~h}$, were suspended in the upper chamber containing $200 \mu \mathrm{l}$ serum-free RPMI-1640 with the same concentration of the drug. The lower chamber contained $600 \mu \mathrm{l}$ RPMI-1640 supplemented with $10 \%$ NCS containing the same concentration of the drug. After $20 \mathrm{~h}$ incubation, the ES-2 or $3 \mathrm{AO}$ cells remaining on the upper surface of the filters were removed by wiping the surface with cotton swabs. The migrated cells on the lower surface of the filters were fixed with methanol (Laiyang Fine Chemical Factory, Yantai, China) for $10 \mathrm{~min}$ and then stained with $2 \mathrm{mg} / \mathrm{ml}$ crystal violet dye (Kermel, Tianjin, China) for $10 \mathrm{~min}$. Images of the migrated cells were captured under a microscope (IX81; Olympus, Tokyo, Japan) and the results are presented as the mean number of migrated cells observed in five different fields.

In vitro invasion assay. An in vitro invasion assay was performed using invasion assays, as described previously (3). Matrigel was diluted 1:19 with serum-free RPMI-1640. The dilution $(50 \mu \mathrm{l})$ was used to coat the upper transwell chamber for $1.5 \mathrm{~h}$. The same cell migration assay was then conducted on the ES-2 and $3 \mathrm{AO}$ cells.

Assessment of $\mathrm{DH}-12 \mathrm{a}$ anti-tumour activity in vivo. This assay was performed according to previously described methods (16). In brief, a xenograft mouse model was established by subcutaneously inoculating $100 \mu 17.6 \times 10^{6} \mathrm{H} 22$ cells in the right armpit of 5-week-old female Kunming mice following adaptation for 1 week. For inoculation, the H22 cells were washed three times with sterile PBS and re-suspended in sterile PBS. Following growing for 5 days, any mice which were under or overweight were excluded and the remaining mice were randomly divided into four groups. For the drug treatment, the drugs (Bestatin, DH-12a and 5-Fu) dissolved in sterile PBS with 5\% DMSO were administered by intraperitoneal injection for 5 days and withdrawn for 2 days. This procedure was performed in two courses for 14 days. Each group of mice was treated with one of the following: i) 5-Fu (30 mg/kg/day in the first course and $25 \mathrm{mg} / \mathrm{kg} /$ day in the second course for evident weight loss); ii) 5-Fu and Bestatin (20 mg/kg/day each); iii) 5-Fu and DH-12a (20 mg/kg/day each) and iv) PBS with 5\% DMSO. These treatments were administered at a volume of $200 \mu \mathrm{l} / 20 \mathrm{~g}$. The mice were then sacrificed and the liver, spleen and tumour of each mouse were weighed for statistical analysis.

Lung tumour metastasis assay. $\mathrm{H} 22$ cells $\left(7.5 \times 10^{6}\right)$ in $200 \mu \mathrm{l}$ sterile PBS were injected into 4-6-week-old female Kunming mice by tail vein injection. After $24 \mathrm{~h}$, the mice were randomly divided into four groups (17). For the drug treatment, the drugs, dissolved in sterile PBS with 5\% DMSO, were administered by intraperitoneal injection for 5 days and withdrawn for 2 days. This procedure was performed in two courses for 14 days. Each group of mice was treated with one of the following: i) 5 -Fu $(26.72 \mathrm{mg} / \mathrm{kg})$; ii) $5-\mathrm{Fu}(26.72 \mathrm{mg} / \mathrm{kg})$ and Bestatin $(63.34 \mathrm{mg} / \mathrm{kg})$; iii) 5-Fu $(26.72 \mathrm{mg} / \mathrm{kg})$ and $\mathrm{DH}-12 \mathrm{a}$ $(67.15 \mathrm{mg} / \mathrm{kg})$ and iv) PBS with 5\% DMSO. These treatments were administered at a volume of $200 \mu \mathrm{l} / 20 \mathrm{~g}$. The mice were then sacrificed and the lung, liver and spleen of each mouse were weighed for statistical analysis. Lung tissues were then fixed with Bouin (Hede Biotechnology Co., Ltd., Beijing, China) and the nodes on each lung surface were counted. Single factor analysis of variance (ANOVA) with Origin 7.5 software (OriginLab Corporation, Northanpton, MA, USA) was used to calculate the overall difference.

Statistical analysis. The data are expressed as the mean \pm standard deviation. Statistical analysis was performed by one-way ANOVA. $\mathrm{P}<0.05$ was considered to indicate a statistically significant difference.

\section{Results}

Determination of APN expression. As shown in Fig. 2, FACS analysis revealed that the ES-2 cell line was positive for APN while the $3 \mathrm{AO}$ cell line was negative for APN. This result provided the basis for using ES-2 and $3 \mathrm{AO}$ cells to determine the biological activity of DH-12a as an APN inhibitor.

Suppression of APN activity. As shown in Fig. 3, DH-12a and Bestatin decreased the activity of APN in ES-2 cells in a dose-dependent manner. At concentrations between 2.5 and $640 \mu \mathrm{M}$, the mean inhibition rates of DH-12a on the activity of APN were significantly higher than that of Bestatin. The mean inhibition rates of $\mathrm{DH}-12 \mathrm{a}$ and Bestatin were 28.9 and $19.9 \%$ at $2.5 \mu \mathrm{M}$ and 88.9 and $81.6 \%$ at $640 \mu \mathrm{M}$, respectively.

APN inhibition constants. Following construction of the standard curves of $1 / v$ vs. [I] at different substrate concentrations, the $\mathrm{K}_{\mathrm{i}}$ of DH-12a and Bestatin was determined as -[I] at the point of intersection (Fig. 4). For DH-12a, $\mathrm{K}_{\mathrm{i}}=13.15 \mu \mathrm{M}$; for Bestatin $\mathrm{K}_{\mathrm{i}}=16.57 \mu \mathrm{M}$.

Inhibition of cell proliferation. As shown in Fig. 5, DH-12a and Bestatin elicited a dose-dependent effect on the four cell lines after $48 \mathrm{~h}$ exposure. In the ES-2 cells (Fig. 5A), the maximum inhibition rates were $58.5 \%$ at $160 \mu \mathrm{M} \mathrm{DH}-12 \mathrm{a}\left(\mathrm{IC}_{50}=43.8 \mu \mathrm{M}\right)$ and $34.7 \%$ at $640 \mu \mathrm{M}$ Bestatin. In the $3 \mathrm{AO}$ cells (Fig. 5B), the maximum inhibition rates were $53.9 \%$ at $160 \mu \mathrm{M} \mathrm{DH}-12 \mathrm{a}$ $\left(\mathrm{IC}_{50}=70.2 \mu \mathrm{M}\right)$ and $32.4 \%$ at $640 \mu \mathrm{M}$ Bestatin. In addition, at the same concentration, Bestatin exhibited a higher inhibitory rate in the $3 \mathrm{AO}$ cells than in the ES- 2 cells, while Bestatin is an APN inhibitor. As Fig. 5C and D show, in the HL60 and A549 cells, the inhibitory rates of $\mathrm{DH}-12 \mathrm{a}$ and Bestatin decreased more significantly than in the ES-2 and $3 \mathrm{AO}$ cells.

Decrease in cell migration. APN is also involved in tumour cell migration $(18,19)$. By comparing the blank control groups, the present study found that the number of $3 \mathrm{AO}$ cells that migrated through the filter was markedly lower than that of the ES-2 cells. As shown in Fig. 6, in ES-2 cells, the inhibitory rates on cell migration at $15 \mu \mathrm{M} \mathrm{DH}-12 \mathrm{a}$ and $320 \mu \mathrm{M}$ Bestatin were 56.4 and $22.3 \%$, respectively. In $3 \mathrm{AO}$ cells, the inhibitory rates at $15 \mu \mathrm{M}$ DH-12a and $320 \mu \mathrm{M}$ Bestatin were 76.5 and $22.2 \%$, respectively.

Decrease in ES-2 and $3 A O$ cell invasion. The blank control groups demonstrated a significantly higher number of ES-2 

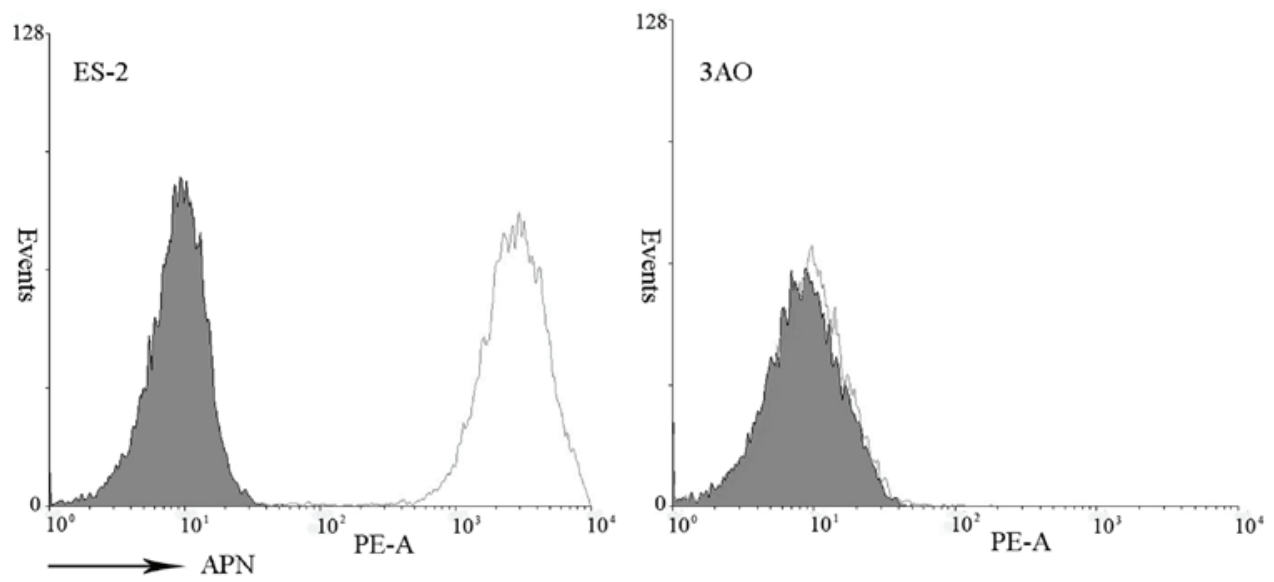

Figure 2. FACS analysis of APN expression in ES-2 and 3AO cell lines. Cells were incubated with phycoerythrin-conjugated monoclonal antibody specific for APN and analysed using FACScan. FACS, fluorescence-activated cell sorting; APN, aminopeptidase N.

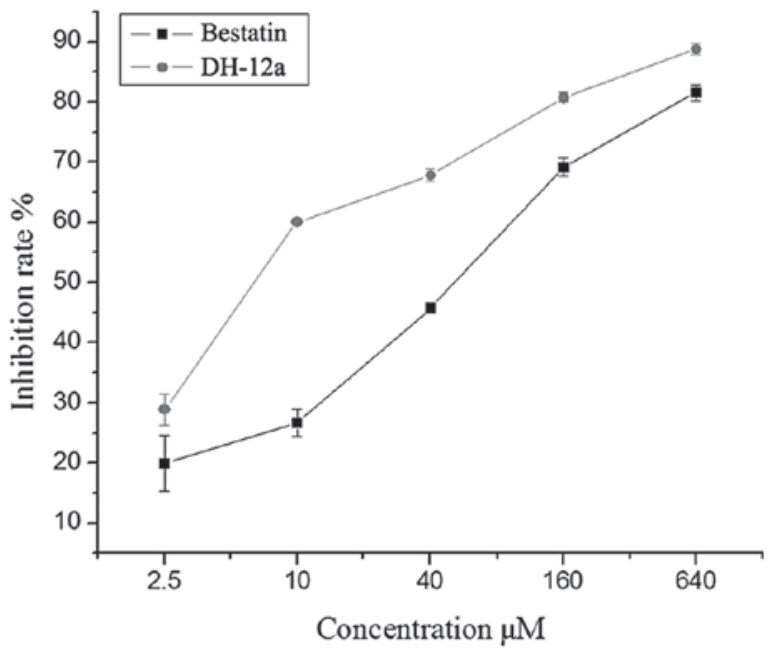

Figure 3. Effects of Bestatin and DH-12a on the enzyme activity of aminopeptidase $\mathrm{N}$ in ES-2 cells. Enzyme activity was estimated by determining the absorbance at $405 \mathrm{~nm}$ and using L-leucine-p-nitroanilide as the substrate. Bars represent the mean \pm standard deviation $(\mathrm{n}=3)$.

cells that had migrated through the matrix than in the $3 \mathrm{AO}$ cells (data not shown). In the ES-2 cells, the inhibitory rates of DH-12a and Bestatin were 75.6 and 43.9\%, respectively (Fig. 7), whereas in the $3 \mathrm{AO}$ cells, the inhibitory rates of DH-12a and Bestatin were 66.5 and $51.4 \%$, respectively.

Tumour inhibition in vivo. Among the four groups, only the group treated with Bestatin combined with 5-Fu successfully yielded two non-tumourigenic mice. The other groups comprised tumourigenic mice. As shown in Fig. 8, the mean tumour weights of the groups treated with DH-12a combined with 5-Fu, Bestatin combined with 5-Fu and 5-Fu alone were $0.59,0.90$ and $0.85 \mathrm{~g}$, respectively. The mean tumour weight in the blank group was $2.76 \mathrm{~g}$. In addition, no weight loss or increased quality of the liver or spleen was observed in the three drug-treated groups at the end of the experiment.

Depression of lung tumour metastasis. As shown in Fig. 9, the mean number of nodules in the groups treated with DH-12a
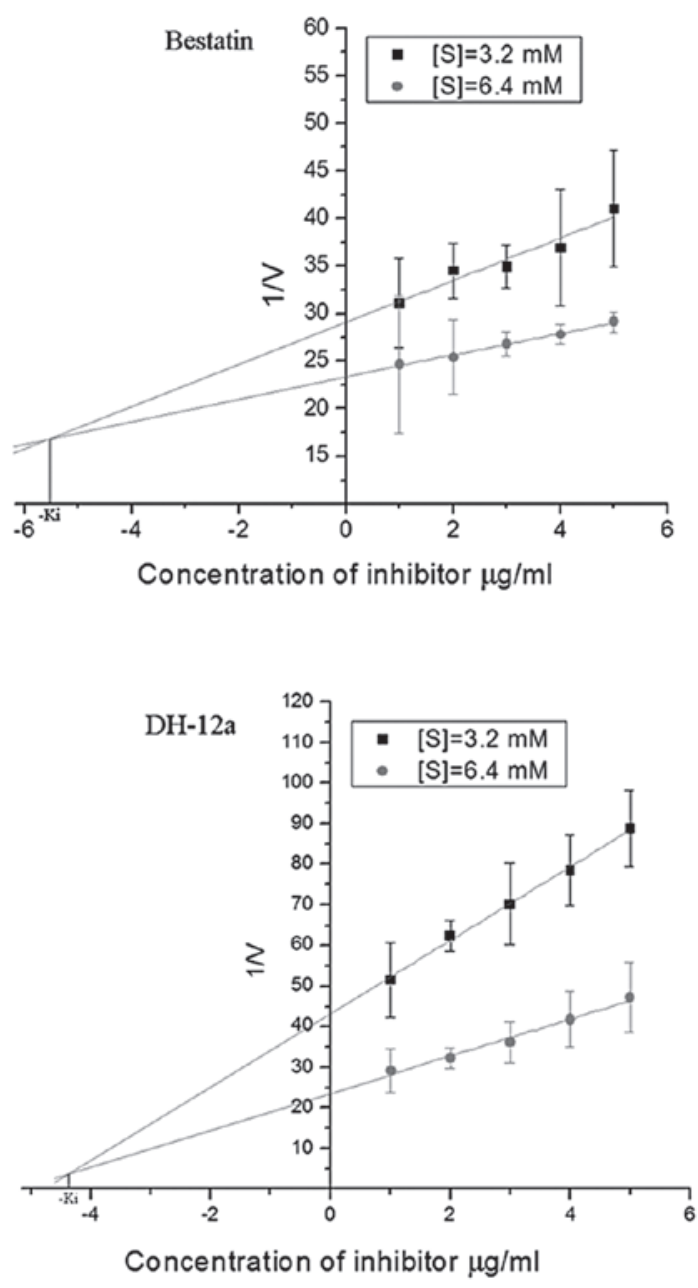

Figure 4. Determination of $\mathrm{K}_{\mathrm{i}}$. The $\mathrm{K}_{\mathrm{i}}$ of Bestatin and DH-12a were determined using Dixon's method, in which ES-2 cells were used as the aminopeptidase $\mathrm{N}$ source. Bars represent the mean \pm standard deviation $(\mathrm{n}=3)$. $\mathrm{K}_{\mathrm{i}}$, inhibition constant.

combined with 5-Fu, Bestatin combined with 5-Fu and 5-Fu alone were $20.5,17.8$ and 23.2 , respectively and the mean number in the blank group was 49.6. The group treated with 
A

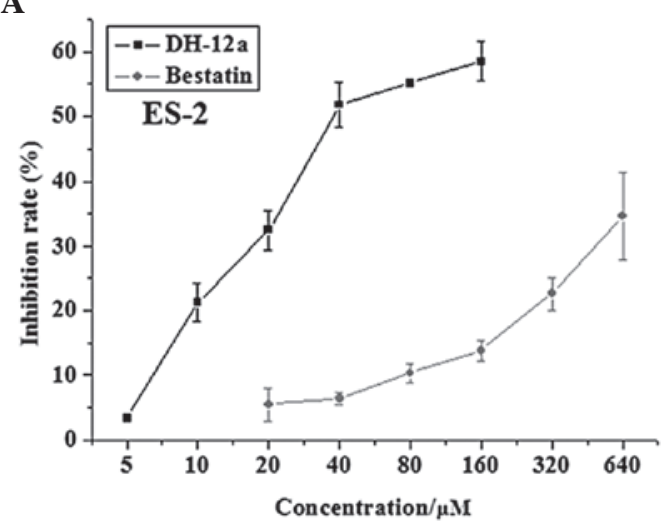

C

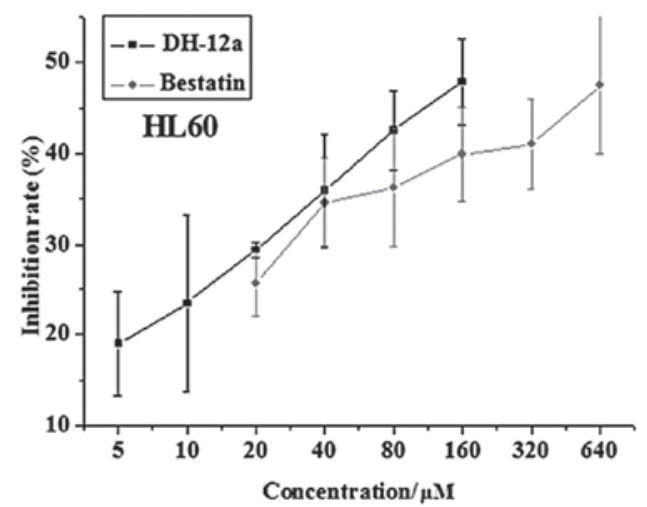

B

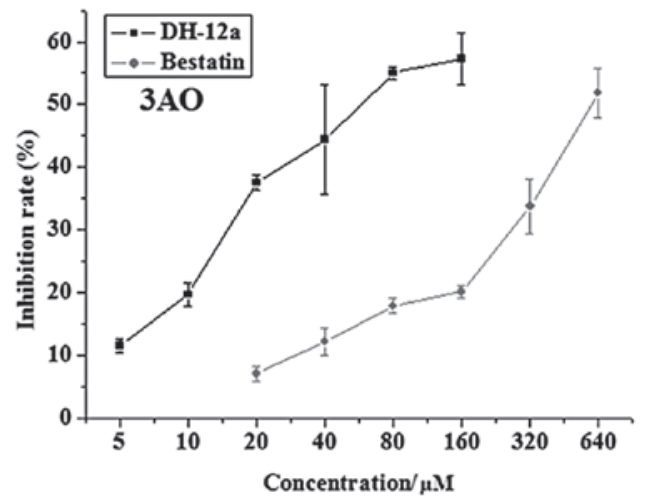

D

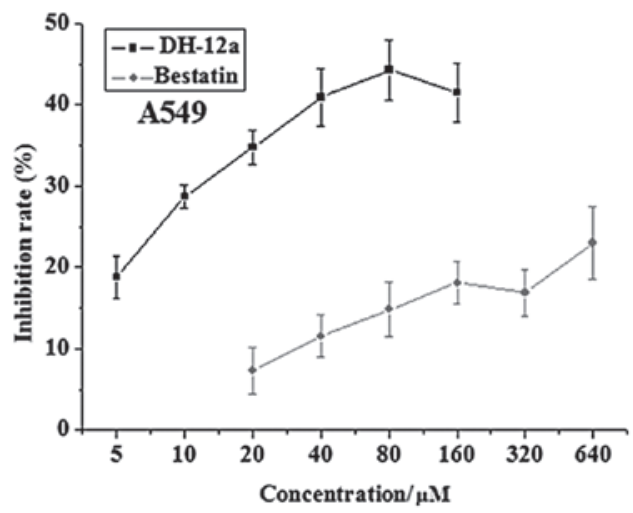

Figure 5. Effects of Bestatin and DH-12a on cell viability determined using an MTT assay. The inhibition rate was calculated according to the following equation: Inhibition rate $=($ ODcontrol - ODtested $) /$ ODcontrol x 100\%. Bars represent the mean \pm standard deviation $(n=3)$. OD, optical density.
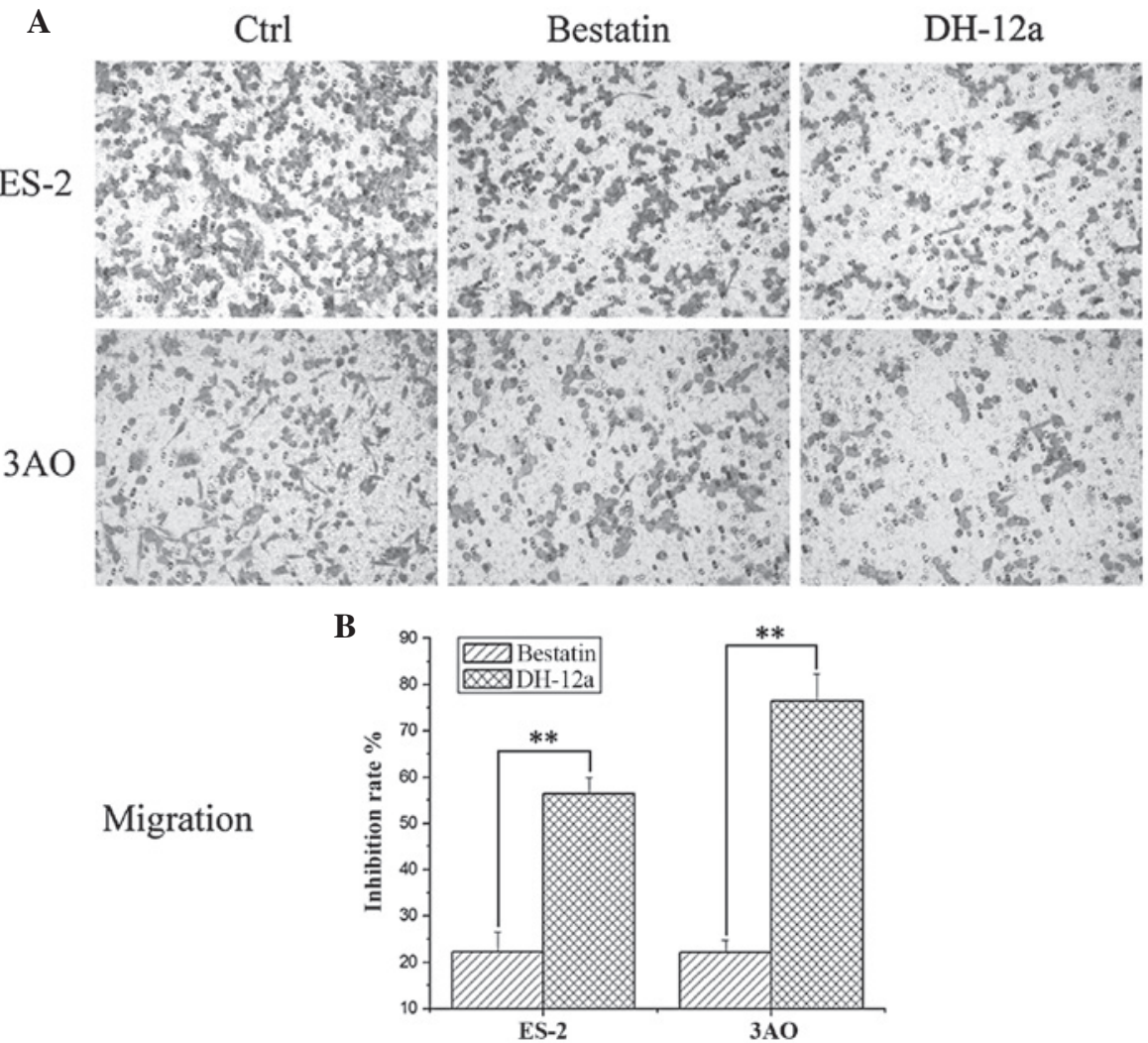

Figure 6. Effects of Bestatin and DH-12a on cell migration determined using an in vitro migration assay. ES-2 and 3AO cells, treated with different compounds, were plated in Matrigel-free transwell chambers and (A) images were captured after $20 \mathrm{~h}$ (magnification, x100). Data in (B) represent the mean number of invading cells from five different fields. ${ }^{* *} \mathrm{P}<0.01$. Ctrl, control. 

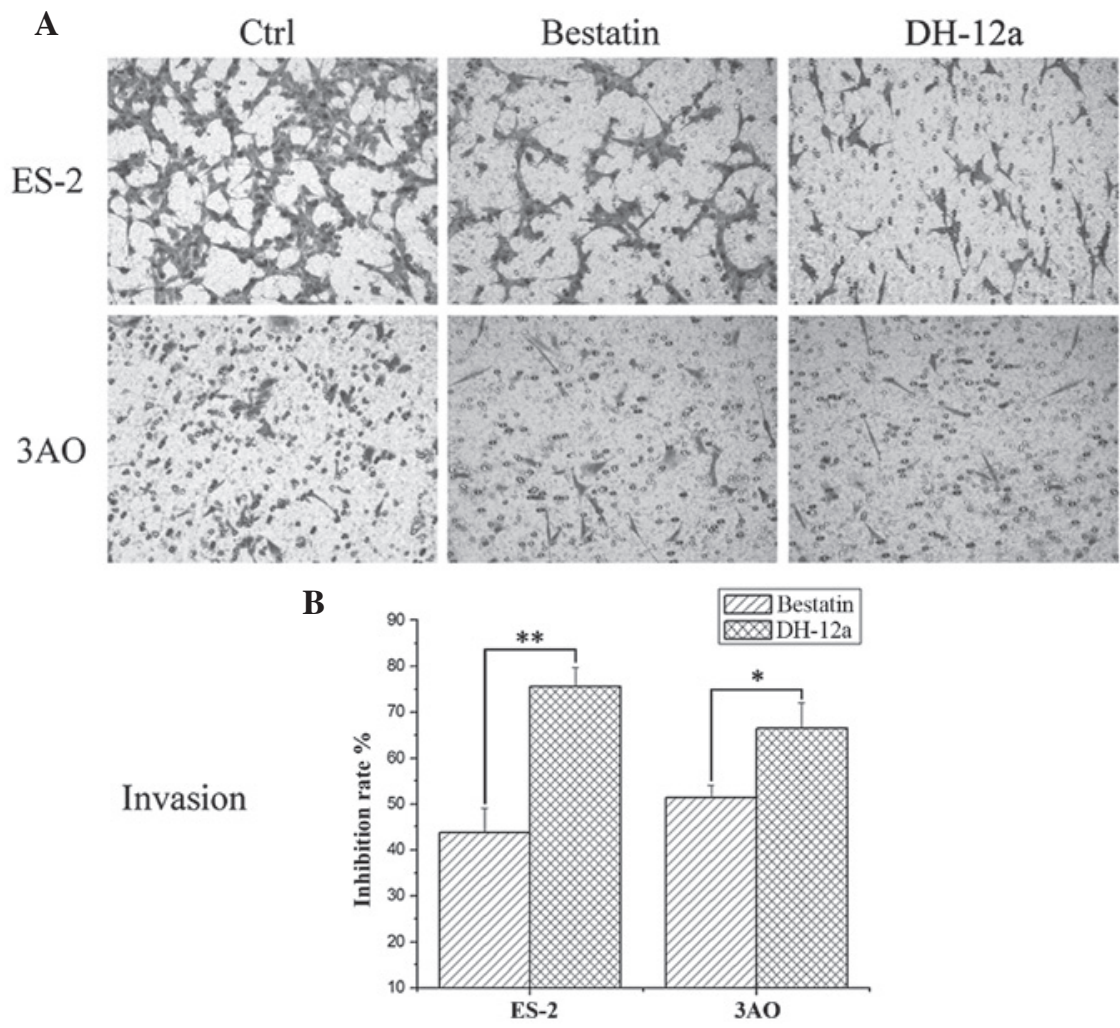

Figure 7. Effects of Bestatin and DH-12a on cell invasion determined using an in vitro invasion assay. ES-2 and $3 \mathrm{AO}$ cells, treated with different compounds, were plated in Matrigel-coated transwell chambers and (A) images were captured after $24 \mathrm{~h}$ (magnification, x100). (B) Data represent the mean number of invading cells from five fields. ${ }^{*} \mathrm{P}<0.05,{ }^{* *} \mathrm{P}<0.01$. Ctrl, control.
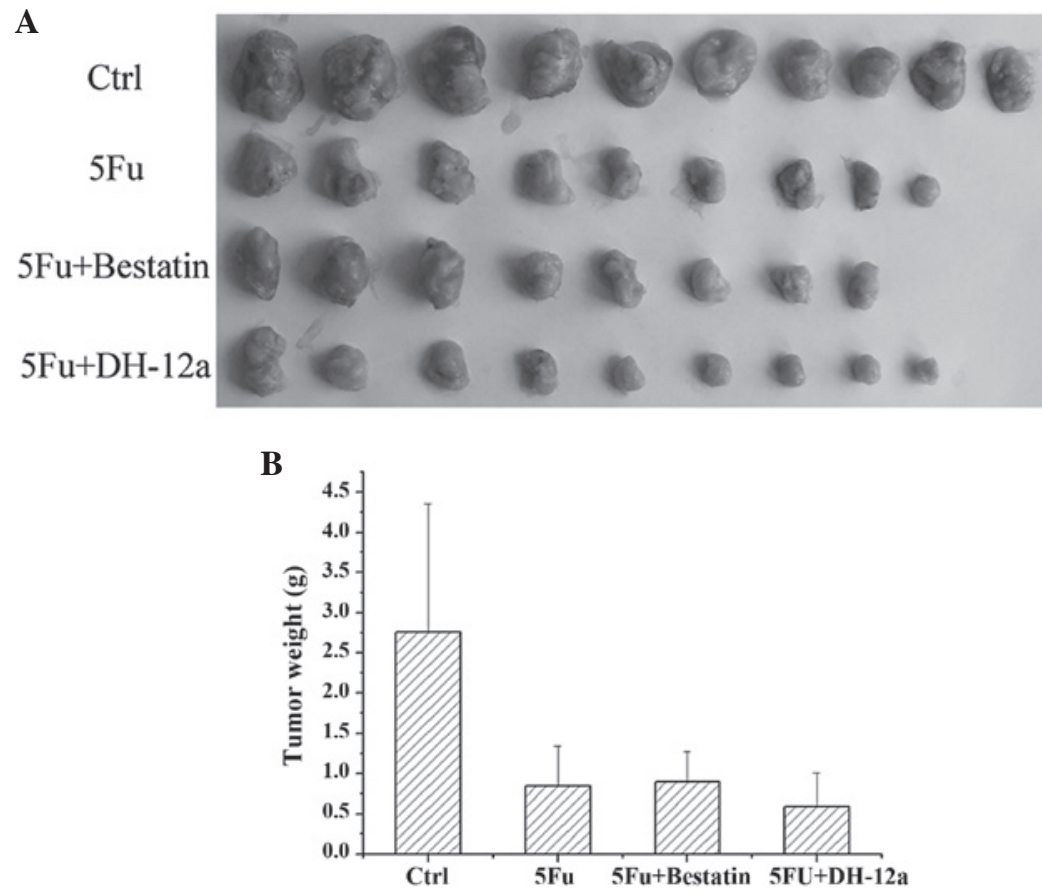

Figure 8. Mean tumour weights of the mice treated with different compounds. The (A) tumours of the mice were weighed following two courses of drug treatment. (B) Bars represent the means of each group. Ctrl, control; 5-Fu, 5-fluorouracil.

Bestatin combined with 5-Fu and the blank group had a standard deviation $>20$. In addition, no weight loss or increased quality in liver or spleen was observed in the three drug-treated groups at the end of the experiment.

\section{Discussion}

Previous studies have revealed that APN is involved in enhanced cell viability, cell migration and tumour inva- 
A

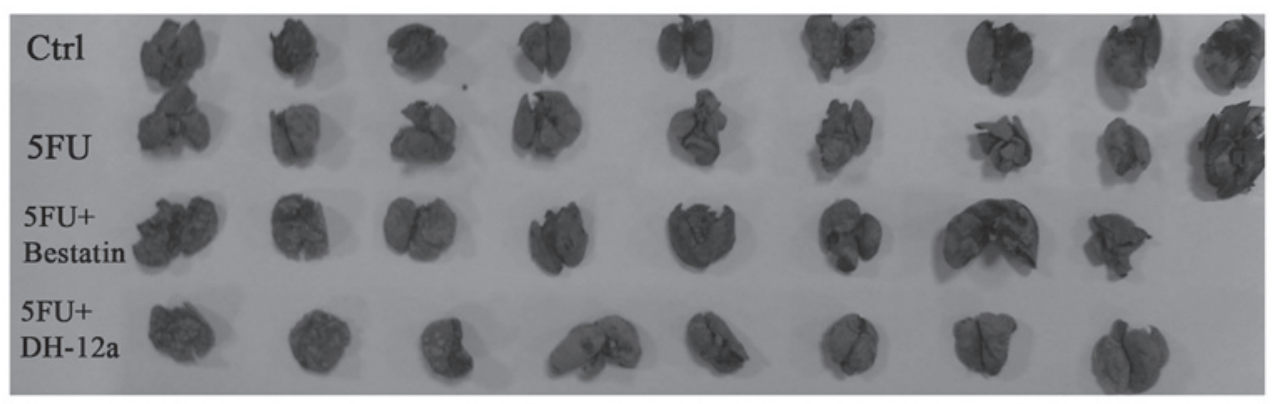

B

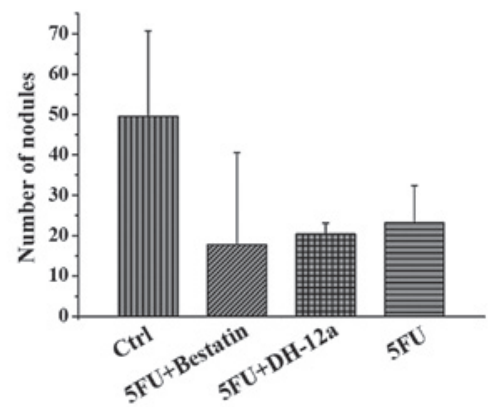

Figure 9. Mean nodules of mice treated with different compounds. (A) Nodules of the mice were counted following two courses of drug treatment. (B) Bars represent the means of each group. Ctrl, control; 5-Fu, 5-fluorouracil.

sion (20-24). In the present study, the results from FACS analysis demonstrated that ES-2 cells markedly expressed APN. This result was consistent with the result of a previous study (3). By contrast, the $3 \mathrm{AO}$ cells were negative for APN. These results were favorable for determining the anti-tumour activity of DH-12a as an APN inhibitor. The result of the enzyme activity assay demonstrated that inhibition of APN activity induced by DH-12a was significantly higher than that by Bestatin at the same concentration. The result of the inhibition constant assay demonstrated that DH-12a yielded a smaller APN inhibition constant than Bestatin, indicating that DH-12a had a stronger APN binding capacity than Bestatin. Therefore, DH-12a was a stronger inhibitor of APN than Bestatin.

Considering that expression of APN in OVCA cells stimulates cell proliferation and tumour invasion (21), the present study determined the effects of DH-12a on cell viability, cell migration and invasion of the ES-2 and $3 \mathrm{AO}$ cell lines. In these experiments, Bestatin was used as the positive control treatment.

In the MTT assay, HL60 and A549 cell lines, which have been demonstrated to have a high expression of APN, were involved. The results demonstrated that the proliferation of ES-2 and 3AO cells were markedly inhibited by DH-12a and the inhibition by $\mathrm{DH}-12 \mathrm{a}$ was significantly higher than that of Bestatin. Notably, no difference in the inhibition of cell viability by the two drugs was observed between the ES-2 and 3AO cells. In the HL60 and A549 cell lines, the inhibitory rates declined compared with those of the ES-2 or $3 \mathrm{AO}$ cell lines, which indicated that DH-12a and Bestatin, as APN inhibiting drugs, had specificity for OVCA cell lines. This was consistent with the application of Bestatin in clinical tumour treatment. In all cell lines, DH-12a persistently exhibited a stronger anti-tumour activity than Bestatin.

The present study also investigated the effect of DH-12a on cell migration and invasion in the ES-2 and 3AO cell lines using a transwell plate assay. In these two assays, the migratory ability and invasive ability of the $3 \mathrm{AO}$ cells were significantly weaker than those of the ES-2 cells. This weak ability may be attributed to the low expression of APN in 3AO cells. These findings are consistent with previous studies, in which APN was reported to function in tumour angiogenesis and tumour invasion $(12,25)$. In these two assays, DH-12a exhibited persistently and significantly higher inhibitory rates than Bestatin at the same concentration, suggesting that $\mathrm{DH}-12 \mathrm{a}$ was able to inhibit cell migration or invasion in the OVCA cell lines to a greater extent than Bestatin. However, in 3AO and ES-2 cell lines, neither DH-12a nor Bestatin demonstrated an increased inhibitory rate in the ES-2 cell line, suggesting that APN inhibitors have other targets in the OVCA cell lines. In addition, DH-12a is possibly a non-specific APN inhibitor exhibiting a more marked anti-tumour activity than Bestatin.

All the in vitro assays indicated that DH-12a exerted a superior anti-tumour ability compared with Bestatin, and that inhibition of the in vitro cell migration and invasion of the $3 \mathrm{AO}$ cell line, which was APN negative, suggested that other targets in the OVCA cell lines were important in the progress of the $3 \mathrm{AO}$ and ES-2 cell lines.

The in vivo assay revealed that $5-\mathrm{Fu}$, Bestatin combined with 5-Fu and DH-12a combined with 5-Fu markedly inhibited tumour growth and lung tumour metastasis without exhibiting evident biological toxicity. Tumour inhibition was most marked in the group treated with $\mathrm{DH}-12$ a combined with $5-\mathrm{Fu}$, suggesting that DH-12a exhibited more effective anti-tumour activity than Bestatin. DH-12a may reduce the toxicity and enhance the efficacy of treatment compared with genotoxic chemotherapeutic 5-Fu alone.

In conclusion, DH-12a exhibited a stronger ability in inhibiting cancer cell proliferation than Bestatin in the four cell lines. The ability of DH-12a to inhibit in vitro cell migration and cell invasion was also significantly higher than Bestatin in the $3 \mathrm{AO}$ and ES-2 cell lines. In vivo, DH-12a exhibited good biocompatibility in mice and its anti-tumour ability was higher than that of Bestatin. In conclusion, as an initial investigation of 
potential drugs for inhibition of APN, the present study provided encouraging results and thus further investigation of DH-12a for clinical application is required.

\section{Acknowledgements}

This study was supported by the Weifang High-tech Zone Biomedical Science and Technology Industrial Park, the Shandong Province Outstanding Youth Scientist Foundation Plan (grant no. BS2013YY020), the Shandong Medical and Health Science and Technology Development Plan (grant no. 2013WS0282), the Natural Science Foundation of Shandong Province (grant no. ZR2010DM010) and the Higher Educational Science and Technology Program of Shandong Province (grant no. J10LC20).

\section{References}

1. Hooper NM: Families of zinc metalloproteases. FEBS Lett 354 1-6, 1994.

2. Look AT, Ashmun RA, Shapiro LH and Peiper SC: Human myeloid plasma membrane glycoprotein CD13 (gp150) is identical to aminopeptidase N. J Clin Invest 83: 1299-1307, 1989.

3. Wang X, Jing F, Zhu H, Fang H, Zhang J and Xu W: Activity screening and structure-activity relationship of the hit compounds targeting APN/CD13. Fundam Clin Pharmacol 25: 217-228, 2011.

4. Ishii K, Usui S, Sugimura Y, Yoshida S, Hioki T, Tatematsu M, Yamamoto $\mathrm{H}$ and Hirano $\mathrm{K}$ : Aminopeptidase $\mathrm{N}$ regulated by zinc in human prostate participates in tumor cell invasion. Int J Cancer 92: 49-54, 2001.

5. Ishii K, Usui S, Sugimura Y, Yamamoto H, Yoshikawa K and Hirano K: Inhibition of aminopeptidase N (AP-N) and urokinase-type plasminogen activator (uPA) by zinc suppresses the invasion activity in human urological cancer cells. Biol Pharm Bull 24: 226-230, 2001

6. Tokuhara T, Hattori N, Ishida H, Hirai T, Higashiyama M, Kodama K and Miyake M: Clinical significance of aminopeptidase $\mathrm{N}$ in non-small cell lung cancer. Clin Cancer Res 12: 3971-3978, 2006.

7. Ikeda N, Nakajima Y, Tokuhara T, Hattori N, Sho M, Kanehiro H and Miyake M: Clinical significance of aminopeptidase N/CD13 expression in human pancreatic carcinoma. Clin Cancer Res 9: $1503-1508,2003$

8. Wang C, Fan G, Lin M, Chen Y, Zhao W and Wu Y: Development of a liquid chromatography/tandem mass spectrometry assay for the determination of bestatin in rat plasma and its application to a pharmacokinetic study. J Chromatogr B Analyt Technol Biomed Life Sci 850: 101-108, 2007.

9. Yamashita M, Kajiyama H, Terauchi M, Shibata K, Ino K, Nawa A, Mizutani S and Kikkawa F: Involvement of aminopeptidase $\mathrm{N}$ in enhanced chemosensitivity to paclitaxel in ovarian carcinoma in vitro and in vivo. Int J Cancer 120: 2243-2250, 2007.

10. Tsukamoto H, Shibata K, Kajiyama H, Terauchi M, Nawa A and Kikkawa F: Aminopeptidase N (APN)/CD13 inhibitor, Ubenimex, enhances radiation sensitivity in human cervical cancer. BMC Cancer 8: 74-81, 2008.
11. Sakuraya M, Tamura J, Itoh K, Kubota $K$ and Naruse T: Aminopeptidase inhibitor ubenimex inhibits the growth of leukaemic cell lines and myeloma cells through its cytotoxicity. J Int Med Res 28: 214-221, 2000.

12. Jin K, Zhang X, Ma C, Xu Y, Yuan Y and Xu W: Novel indoline-2,3-dione derivatives as inhibitors of aminopeptidase N (APN). Bioorg Med Chem 21: 2663-2670, 2012.

13. Terauchi M, Kajiyama H, Shibata K, Ino K, Nawa A, Mizutani S and Kikkawa F: Inhibition of APN/CD13 leads to suppressed progressive potential in ovarian carcinoma cells. BMC Cancer 7: 140, 2007.

14. Qu XJ, Yang JL, Russell PJ and Goldstein D: Changes in epidermal growth factor receptor expression in human bladder cancer cell lines following interferon-alpha treatment. J Urol 172: 733-738, 2004.

15. Zhang P, Chen JH, Dong X, Tang MT, Gao LY, Zhao GS, Yu LG and Guo XL: 6r, a novel oxadiazole analogue of ethacrynic acid, exhibits antitumor activity both in vitro and in vivo by induction of cell apoptosis and S-phase arrest. Biomed Pharmacother 67: $58-65,2013$.

16. Haraguchi N, Ishii H, Mimori K, Tanaka F, Ohkuma M, Kim HM, Akita H, Takiuchi D, Hatano H, Nagano $\mathrm{H}$, Barnard GF, Doki Y and Mori M: CD13 is a therapeutic target in human liver cancer stem cells. J Clin Invest 120: 3326-3339, 2010.

17. Jiang WG, Lu XA, Shang BY, Fu Y, Zhang SH, Zhou D, Li L, Li Y, Luo Y and Zhen YS: Genetically engineered endostatin-lidamycin fusion proteins effectively inhibit tumor growth and metastasis. BMC Cancer 13: 479, 2013.

18. Kido A, Krueger S, Haeckel C and Roessner A: Possible contribution of aminopeptidase N (APN/CD13) to invasive potential enhanced by interleukin- 6 and soluble interleukin- 6 receptor in human osteosarcoma cell lines. Clin Exp Metastasis 17: 857-863, 1999

19. Kido A, Krueger S, Haeckel C and Roessner A: Inhibitory effect of antisense aminopeptidase N (APN/CD13) cDNA transfection on the invasive potential of osteosarcoma cells. Clin Exp Metastasis 20: 585-592, 2003.

20. Kehlen A, Lendeckel U, Dralle H, Langner J and Hoang-Vu C: Biological significance of aminopeptidase N/CD13 in thyroid carcinomas. Cancer Res 63: 8500-8506, 2003.

21. Li Q, Fang H, Wang X, Hu L and Xu W: Novel cyclic-imide peptidomimetics as aminopeptidase $\mathrm{N}$ inhibitors. Design, chemistry and activity evaluation. Part I. Eur J Med Chem 44: 4819-4825, 2009.

22. Bhagwat SV, Petrovic N, Okamoto Y and Shapiro LH: The angiogenic regulator CD13/APN is a transcriptional target of Ras signaling pathways in endothelial morphogenesis. Blood 101: 1818-1826, 2003.

23. Petrovic N, Bhagwat SV, Ratzan WJ, Ostrowski MC and Shapiro LH: CD13/APN transcription is induced by RAS/MAPK-mediated phosphorylation of Ets-2 in activated endothelial cells. J Biol Chem 278: 49358-49368, 2003.

24. Bhagwat SV, Lahdenranta J, Giordano R, Arap W, Pasqualini R and Shapiro LH: CD13/APN is activated by angiogenic signals and is essential for capillary tube formation. Blood 97: 652-659, 2001.

25. Raccor BS, Vogt A, Sikorski RP, Madiraju C, Balachandran R, Montgomery K, Shin Y, Fukui Y, Jung WH, Curran DP and Day BW: Cell-based and biochemical structure-activity analyses of analogs of the microtubule stabilizer dictyostatin. Mol Pharmacol 73: 718-726, 2008. 